\title{
STABLE MAPS INTO FREE $G$-SPACES
}

\author{
J. P. C. GREENLEES
}

\begin{abstract}
In this paper we introduce a systematic method for calculating the group of stable equivariant maps $[X, Y]^{G}[\mathbf{3}, \mathbf{1 8}]$ into a $G$-free space or spectrum $Y$. In fact the method applies without restriction on $X$ whenever $G$ is a $p$-group and $Y$ is $p$-complete and satisfies standard finiteness assumptions. The method is an Adams spectral sequence based on a new equivariant cohomology theory $c^{*}(X)$ which we introduce in $\S 1$. This spectral sequence is quite calculable and provides a natural generalisation of the classical Adams spectral sequence based on ordinary mod $p$ cohomology. It also geometrically realises certain inverse limits of nonequivariant Adams spectral sequences which have been useful in the study of the Segal conjecture $[\mathbf{1 9}, \mathbf{5}, \mathbf{2 1}, \mathbf{9}]$.
\end{abstract}

0. Introduction. In what follows, $G$ is a finite group and we work in a stable equivariant homotopy category, such as that of Lewis-May [18]. All spaces are pointed and all homology and cohomology is reduced.

Our main theorem establishes the power of $\bmod p$ Borel cohomology [6]

$$
b^{*}(X)=H^{*}\left(E G_{+} \wedge_{G} X ; \mathbf{F}_{p}\right)
$$

as an invariant in studying topology with a $p$-group $G$ of equivariance.

THEOREM 0. Suppose that $G$ is a finite p-group and that $Y$ is a $G$-space or spectrum which is

(i) $G$-free,

(ii) $p$-complete,

(iii) bounded below, and

(iv) homologically locally finite (i.e. $H^{i}\left(Y ; \mathbf{F}_{p}\right)$ is finite for every $\left.i\right)$. For any $G$-space or spectrum $X$, there is a convergent Adams spectral sequence

$$
E_{2}^{s, t}=\operatorname{Ext}_{b^{*} b}^{s, t}\left(b^{*} Y, c^{*} X\right) \Rightarrow[X, Y]_{*}^{G} .
$$

Moreover the spectral sequence is natural in both variables.

REMARKS. (1) The cohomology theory $c^{*}(\cdot)$ will be introduced in $\S 1$; it agrees with Borel cohomology on $G$-free spaces or spectra. Therefore the spectral sequence is a generalisation of that based on Borel cohomology alone.

(2) In $\S 2$ we identify the algebra $b^{*} b$ of stable natural transformations of Borel cohomology in familiar terms. Indeed we have an isomorphism (2.7)

$$
b^{*} b \cong H^{*}\left(B G_{+} ; \mathbf{F}_{p}\right) \tilde{\otimes} A^{*}
$$

Received by the editors July $20,1987$.

1980 Mathematics Subject Classification (1985 Revision). Primary 55N91, 55S91, 55T15, 57S17; Secondary $55 \mathrm{~N} 20,55 \mathrm{~N} 25,55 \mathrm{P} 42,55 \mathrm{~S} 37$.

Key words and phrases. Adams spectral sequence, Borel cohomology, equivariant cohomology, Segal conjecture, Singer construction. 
where $A^{*}$ is the usual $\bmod p$ Steenrod algebra $[\mathbf{2 2}, \mathbf{2 6}]$ and $\tilde{\otimes}$ denotes the twisted (or semi-) tensor product in the sense of Massey-Peterson [20]. We will also explain explicitly how this algebra acts on $b^{*}(X)$. Much of this material is due to $\mathrm{J}$. F. Adams and we are grateful to him for permission to include an account of it.

(3) (a) Convergence is in the usual conditional sense of Boardman [5] appropriate to Adams spectral sequences.

(b) Since $Y$ is $G$-free the conditions (ii), (iii) and (iv) need only be satisfied nonequivariantly.

(4) The condition that $Y$ is $G$-free is essential. However we will show elsewhere $[13,14]$ that if we use Borel homology instead of co-Borel cohomology this condition may be considerably weakened (and dropped entirely if $G$ is elementary abelian).

(5) When $G$ is the trivial group this spectral sequence reduces to the classical Adams spectral sequence based on mod $p$ cohomology [1].

(6) The proof of convergence relies fundamentally on Adams' nonequivariant convergence theorem $[\mathbf{1}, \mathbf{2}]$ and on the fact that $\mathbf{F}_{p}$ is the only simple $\mathbf{F}_{p} G$-module when $G$ is a $p$-group (see (4.2)). The other important ingredient is the fact that $b^{*} b$ is a connected $\mathbf{F}_{p}$-algebra; this and the requisite good behaviour necessary for the calculation of the $E_{2}$-term can be traced back to (2.8).

There have been attempts to approach a similar ideal by nonequivariant methods from at least two directions. Firstly various inverse limits of classical Adams spectral sequences have been used $[\mathbf{1 9}, \mathbf{5}, \mathbf{2 1}, \mathbf{9}]$ and are related to our point of view by Adams' isomorphism

$$
[X, Y]^{G}=[X, Y / G]^{1} \quad([\mathbf{3},(5.4)],[\mathbf{1 8},(\text { II. } .2 .2)])
$$

when $X$ is $G$-fixed and $Y$ is $G$-free.

Secondly Adams constructed in unpublished lectures (1983) an Adams spectral sequence based on Borel cohomology alone under restrictive hypotheses. His technique was to reduce to a nonequivariant situation using the isomorphism

$$
[X, Y]^{G}=[X / G, Y]^{1} \quad([\mathbf{3},(5.3)],[\mathbf{1 8},(\mathrm{I} .3 .8)])
$$

when $X$ is $G$-free and $Y$ is $G$-fixed.

We find that by working purely equivariantly we are able to extend the results of both approaches and avoid many technical difficulties.

The rest of the paper is laid out as follows. In $\S 1$ we introduce co-Borel cohomology $c^{*}(X)$, and prove some basic facts about it. In $\S 2$ we give an account of the algebra $b^{*} b$ of all stable operations of Borel cohomology. In $\S 3$ we set up the spectral sequence of Theorem 0 and easily identify the $E_{2}$ term. In $\S 4$ we prove the spectral sequence converges to the required group. Finally, in $\S 5$ we give a method for calculating the $E_{2}$ term of the spectral sequence of Theorem 0 , and use it to show that how the homological properties of $c^{*} X$ and $b^{*} Y$ over $b^{*}=H^{*}\left(B G_{+} ; \mathbf{F}_{p}\right)$ can give rise to geometric consequences generalising certain known results with geometric hypotheses on $X$ and $Y$.

I thank Professor J. F. Adams for suggesting the study of Borel cohomology and for permission to give an account of his unpublished work. I also thank Professor $\mathrm{R}$. Cohen and the referee for suggesting I completely rewrite the previous version of this paper; this obvious course has led to great improvements. 
1. Borel and co-Borel cohomology. In this section we recall the definition of Borel cohomology, introduce a closely related theory and establish their basic properties.

For any virtual real representation $\alpha$ of $G$, let $|\alpha|$ denote its virtual dimension. Recall that Borel cohomology is defined for orientable $\alpha$ by

$$
b^{\alpha}(X ; k)=H^{|\alpha|}\left(E G_{+} \wedge_{G} X ; k\right) .
$$

Suspension isomorphisms for orientable representations come from the Thom maps. We will be mostly concerned with the case that $G$ is a $p$-group and $k=\mathbf{F}_{p}$, when all representations are orientable, but in the general case we refer to [16, 28 and 17] to justify the fact that $b^{\alpha}(X ; k)$ may also be defined for nonorientable $\alpha$. Accordingly Borel cohomology is represented by a $G$-spectrum $b$. Evidently if $\alpha$ is orientable $b^{\alpha}(\cdot ; k)$ is represented for $G$-spaces by the function space $E M(k,|\alpha|)^{E G_{+}}$of pointed maps into a $G$-fixed Eilenberg-Mac Lane space [25].

Henceforth we omit the coefficients $k$, and we use the usual convention that an asterisk refers to $\mathbf{Z}$-gradings and cogradings.

We define the co-Borel spectrum $c$ by $c:=b \wedge E G_{+}$and co-Borel homology and cohomology are the theories represented by it [29]. We have remarked elsewhere that it also admits a chain description [12]. We let $t$ be the Tate spectrum defined by $t:=b \wedge S^{0} * E G$, and recall [11] that it represents Swan's Tate homology and cohomology of $G$-spaces [27]. It is clear that $c$ and $t$ are $b$-modules and hence that $c^{*}(X)$ and $t^{*}(X)$ are naturally modules over the algebra $b^{*} b$ of stable operations of Borel cohomology and that $c_{*}(X)$ and $t_{*}(X)$ are modules over the coefficient ring $b_{*}$. We may now summarise the basic properties of the co-Borel theories.

THEOREM (1.1). (a) (Coefficient module) $c_{*}=H_{*}\left(B G_{+}\right)$.

(b) (Homology theory): $c_{*}(X)=H_{*}\left(E G_{+} \wedge_{G} X\right)$, and the $b^{*}$-module structure comes from cap product with elements of $b^{*}=H^{*}\left(B G_{+}\right)$.

(c) (Cohomology theory): (i) If $X$ is $G$-free we have isomorphisms $c^{*}(X)=$ $b^{*}(X)=H^{*}\left(E G_{+} \wedge_{G} X\right)$.

(ii) If $X$ is nonequivariantly contractible we have the natural isomorphism $c^{*}(X)$ $=t^{*}(X)$.

(d) (Exact sequences): We have the following natural long exact sequences

$$
\begin{aligned}
& \cdots \rightarrow c^{*}(X) \rightarrow b^{*}(X) \rightarrow t^{*}(X) \rightarrow c^{*}\left(S^{-1} X\right) \rightarrow \cdots, \\
& \cdots \rightarrow c_{*}(X) \rightarrow b_{*}(X) \rightarrow t_{*}(X) \rightarrow c_{*}(S X) \rightarrow \cdots
\end{aligned}
$$

PROOF. Part (d) follows from the fundamental cofibering

$$
E G_{+} \rightarrow S^{0} \rightarrow S^{0} * E G
$$

by smashing with $b$. Part (c) then follows from this since Tate cohomology vanishes on free spaces and Borel cohomology vanishes on nonequivariantly contractible spaces.

Part (a) is a special case of part (b), so it remains to prove part (b).

It is clear that Tate homology also vanishes on free spaces, and hence that $c_{*}(X)=b_{*}(X)$ when $X$ is free. In general we find that $c_{*}(X)=c_{*}\left(E G_{+} \wedge X\right)=$ $b_{*}\left(E G_{+} \wedge X\right)$ naturally. 
Now if $X$ is finite and free its Spanier-Whitehead dual is also finite and free, and the duality may be taken with respect to an integer suspension. Hence the object $(D X) / G$ is defined, and we have the equivalence

$$
D(X / G) \simeq(D X) / G \quad([\mathbf{3},(8.5)],[\mathbf{1 8},(\mathrm{III} .2 .13)]) .
$$

Thus

$$
c_{*}(X)=b_{*}(X)=b^{*}(D X)=H^{*}(D X / G)=H_{*}(X / G) .
$$

Furthermore all isomorphisms are natural for stable maps of finite free spectra. The first statement of part (b) follows.

For the remark about cap products it is sufficient to take $X$ finite and free (by finiteness of support for homology). But it is well known that cup and cap product correspond under nonequivariant Spanier-Whitehead duality.

REMARK. It bears endless repetition that Borel and. co-Borel theories are definitely distinct. Indeed the coefficient ring $b_{*}$ is nonzero in negative degrees whilst $c_{*}$ is nonzero in positive degrees.

Indeed for our purposes this property is decisive.

COROLlARY 1.2. The spectrum $c$ is $(-1)$-connected. Moreover if $k$ is a finitely generated abelian group, $c$ may be constructed using finitely many free G-cells in each nonnegative dimension.

We will also need the following corollary of $(1.1)(\mathrm{c})(\mathrm{i})$.

COROLlaRY 1.3. We have the isomorphisms

$$
c^{*} c=b^{*} c=b^{*} b .
$$

Henceforth we restrict attention to $\bmod p$ coefficients, so that $k=\mathbf{F}_{p}$.

2. Stable operations of $\bmod p$ Borel cohomology. In this section we give an account of the algebra of operations of $\bmod p$ Borel cohomology stable for arbitrary representations. We suppose that $p=2$ if $G$ is of even order. First we construct various operations and explain their algebraic behaviour. This is due to J. F. Adams (unpublished lectures 1983) and I am grateful to him for permission to include an account. Finally we include a short calculation to show that all operations occur in this way.

The operations come from two sources: multiplication by elements of the coefficient ring $b^{*}=H^{*}\left(B G_{+}\right)$, and from the nonequivariant Steenrod operations. However we must take care to guarantee stability under all suspensions, and for this we need some notation. Indeed, for each isomorphism class of simple real representations we choose a representative $V$ and a generator of $H^{|V|}\left(S^{V}\right)$; hence we obtain a generator $\tau_{V} \in H^{|V|}\left(E G \times_{G} S^{V}, E G \times_{G} \infty\right)=b^{[V]}\left(S^{V}\right)$. From this we define the suspension isomorphisms

$$
\sigma_{V}: b^{\alpha}(X) \rightarrow b^{\alpha+[V]}\left(S^{V} \wedge X\right)
$$

by

$$
\sigma_{V}(x)=\tau_{V} \cdot p^{*} x
$$

where $p$ is the projection map

$$
\begin{aligned}
p:\left(E G \times_{G}\left(S^{V} \times X\right), E G \times{ }_{G}\left(S^{V} \times * \cup \infty \times X\right)\right) \\
\rightarrow\left(E G \times \times_{G}(\infty \times X), E G \times \times_{G}(\infty \times *)\right) .
\end{aligned}
$$

We will usually suppress all mention of $p$. 
To define arbitrary suspension isomorphisms we need an ordering of the simple representations so that we have a chosen generator $\tau_{V} \in b^{[V]}\left(S^{V}\right)$ for all representations $V$.

Since suspension isomorphisms are defined in this way the stability of multiplication by elements of $H^{*}\left(B G_{+}\right)$comes from the fact that $H^{*}\left(B G_{+}\right)$is a graded commutative ring and Borel cohomology is naturally a module over it.

We already have a natural operation of the Steenrod algebra on $b^{*}(X)$ but it is only stable under $G$-fixed suspensions. More generally if $a$ is an element of the Steenrod algebra $A^{*}$ and $\alpha \in R O(G)$ we will introduce certain Adams-Wu classes $c(a, \alpha) \in H^{|a|}\left(B G_{+}\right)$and then if $x \in b^{\alpha}(X)$ we define $a \circ x \in b^{\alpha+|a|}(X)$ by

$$
a \circ x:=\sum_{i} c\left(a_{i}^{\prime},-\alpha\right) \cdot\left(a_{i}^{\prime \prime} x\right)
$$

where the diagonal expansion of $a$ is $\psi^{*} a=\sum_{i} a_{i}^{\prime} \otimes a_{i}^{\prime \prime}$ and where $a_{i}^{\prime \prime} x$ means the usual operation of the Steenrod algebra.

LEMMA (2.2). There is a unique element $c(a, \alpha) \in H^{|a|}\left(B G_{+}\right)$additive in a so that

(i) For a representation $V c(a,[V]):=(-1)^{|a||V|} \sigma_{V}^{-1}\left(a \cdot \tau_{V}\right)$ and

(ii) $c(a, \alpha+\beta)=\sum_{i} c\left(a_{i}^{\prime}, \alpha\right) c\left(a_{i}^{\prime \prime}, \beta\right)$.

PROOF. It is convenient to collect together the elements $c(a, \alpha)$ for various $a$ and let $c(\alpha)$ denote the element $c(\alpha) \in \operatorname{Hom}\left(A^{*}, H^{*} B G_{+}\right)$with the property that $a\{c(\alpha)\}=c(a, \alpha)$ for every $a$.

The statement (ii) then amounts to the statement that $c(\alpha+\beta)=c(\alpha) c(\beta)$, i.e., that $c$ gives a group homomorphism from the additive group $R O(G)$ to the multiplicative group of sequences in $\prod_{d} A_{d} \otimes H^{d}\left(B G_{+}\right)$starting with $1 \otimes 1$.

By the universal property of Grothendieck groups, it therefore suffices to verify that the definition (i) for representations does satisfy the property (ii).

Note first that the class $c(a,[V])$ defined in (i) is independent of the choice of generator $\tau_{V}$. Now we may verify that $c([V]+[W])=c([V]) c([W])$ by the following calculation.

$$
\begin{aligned}
a \tau_{V+W} & =a\left(\tau_{V} \cdot \tau_{W}\right)=\sum_{i} \pm a_{i}^{\prime} \tau_{V} \cdot a_{i}^{\prime \prime} \tau_{W} \\
& =\sum_{i} \pm \sigma_{V}\left(c\left(a_{i}^{\prime},[V]\right)\right) \cdot \sigma_{W}\left(c\left(a_{i}^{\prime \prime},[W]\right)\right) \\
& =\sum_{i} \pm \tau_{V} c\left(a_{i}^{\prime},[V]\right) \tau_{W} c\left(a_{i}^{\prime \prime},[W]\right) \\
& =\sum_{i} \pm \tau_{V} \tau_{W} c\left(a_{i}^{\prime},[V]\right) c\left(a_{i}^{\prime \prime},[W]\right) .
\end{aligned}
$$

EXERCISE. In the above calculation, and those that follow, verify that the signs work out correctly.

Lemma (2.3). For any $a, b \in A^{*}, \alpha \in R O(G)$ we have

$$
c(a b, \alpha)=\sum_{i} c\left(a_{i}^{\prime}, \alpha\right) \cdot\left\{a_{i}^{\prime \prime} c(b, \alpha)\right\} .
$$


PROOF. The lemma is easily verified if $\alpha$ is a representation. For the general case we may suppose $\alpha=[V]-[W]$ and prove the lemma by induction on $|a b|$. The result is trivial if $|a b|=0$.

Now in the notation of $(2.2)$ we have

$$
\begin{aligned}
a\{c(V) b c(V)\} & =(a b) c(V) \quad \text { (by (2.3) for representations) } \\
& =(a b) c(\alpha+W) \\
& =(a b)\{c(\alpha) c(W)\} \quad(\text { by }(2.2))
\end{aligned}
$$

Evidently the term $c(a b, \alpha)=c(a b, \alpha) c(1, W)$ occurs exactly once, on the righthand side of the above equation. Expanding, and rearranging the other terms, one may check that (2.3) holds for $a b$ as required. We leave this to the reader.

COROLLARY (2.4). By using the equation $(2.1)$, that $a \circ x=a\{c(-\alpha) \cdot x\}$ when $x \in b^{\alpha}(X)$

(i) we obtain an action of the Steenrod algebra on $R O(G)$-graded Borel cohomology,

(ii) the action agrees with the usual action on $b^{*}(X)$,

(iii) the operations a० are stable for all suspensions $S^{V}$,

(iv) the action of $A^{*}$ and multiplication by elements of $b^{*}$ are related by

$$
a \circ(h x)=\sum_{i}(-1)^{\left|a_{i}^{\prime \prime}\right||h|}\left(a_{i}^{\prime} h\right)\left(a_{i}^{\prime \prime} \circ x\right) .
$$

Repackaging the above result we obtain the following.

COROllaRY $(2.5)$. The twisted tensor product $[\mathbf{2 0}] b^{*} \tilde{\otimes} A^{*}$ acts on Borel cohomology as an algebra of stable natural transformations.

ProOF OF (2.4). First observe that since Steenrod operations are stable for integer suspensions $c(n)=1$ for any integer $n$. Part (ii) follows, and by (2.2) $c(\alpha+n)=c(\alpha)$.

Now we prove part (i) by calculation.

$$
\begin{aligned}
a \circ(b \circ x) & =a \circ \sum_{i} c\left(b_{i}^{\prime},-\alpha\right) b_{i}^{\prime \prime} x \\
& =\sum_{i, j} \pm c\left(a_{j}^{1},-\alpha\right) a_{j}^{2} c\left(b_{i}^{\prime},-\alpha\right) a_{j}^{3} b_{i}^{\prime \prime} x \\
& =\sum_{j, k} \pm c\left(a_{k}^{\prime} b_{i}^{\prime},-\alpha\right) a_{k}^{\prime \prime} b_{i}^{\prime \prime} x \quad(\text { by }(2.3)) \\
& =(a b) \circ x .
\end{aligned}
$$


Next we turn to part (iii). Indeed we find

$$
\begin{aligned}
a \circ\left(\sigma_{V} x\right) & =a \circ \tau_{V} x=\sum_{i} \pm c\left(a_{i}^{1},-\alpha-V\right) a_{i}^{2} \tau_{V} a_{i}^{3} x \\
& =\sum_{i} \pm c\left(a_{i}^{1},-\alpha-V\right) \tau_{V} c\left(a_{i}^{2}, V\right) a_{i}^{3} x \\
& = \pm \tau_{V} \sum_{i} c\left(a_{i}^{1},-\alpha-V\right) c\left(a_{i}^{2}, V\right) a_{i}^{3} x \\
& = \pm \tau_{V} \sum_{j} c\left(a_{j}^{\prime},-\alpha\right) a_{j}^{\prime \prime} x \quad(\text { by }(2.2)) \\
& =(-1)^{|V||a|} \sigma_{V}(a \circ x) \quad \text { as required. }
\end{aligned}
$$

Finally part (iv) follows by a similar but easier calculation.

We give an example to show that the introduction of the Adams-Wu classes is necessary.

EXAMPLE (2.6). (order 2) Suppose that $G$ is cyclic of order 2, let $\xi$ denote the nontrivial one-dimensional real representation and $t$ denote the generator of $H^{1}\left(B G_{+}\right)$. With this notation, for any $a, b \in \mathbf{Z}$, we have

$$
c\left(\mathrm{Sq}^{i}, a+b \xi\right)=\left(\begin{array}{l}
b \\
i
\end{array}\right) t^{i}
$$

(order $p$ ) Suppose that $G$ is cyclic of odd prime order $p$, let $\eta_{1}, \eta_{2}, \ldots, \eta_{(p-1) / 2}$ denote the nontrivial 2-dimensional real representations, and choose a generator $x$ of $H^{2}\left(B G_{+}\right)$. With this notation we have, for any integers $a, b_{k}$,

$$
\begin{gathered}
c\left(\beta, a+\sum_{k} b_{k} \eta_{k}\right)=0 \text { and } \\
c\left(P^{i}, a+\sum_{k} b_{k} \eta_{k}\right)=\left(\begin{array}{c}
\sum_{k} b_{k} \\
i
\end{array}\right) x^{i(p-1)} .
\end{gathered}
$$

These identifications follow from the cases $c\left(\mathrm{Sq}^{1}, \xi\right)$ and $c\left(P^{1}, \eta_{k}\right)$ using (2.2) and the unstable axiom. These special cases follow from the injectivity of $S^{0} \rightarrow S^{\xi}$ and $S^{0} \rightarrow S^{\eta_{k}}$ in Borel cohomology, which may be seen by considering the cofibre in each case. This proof makes plain that the binomial coefficients are defined for all integers $b$ by $\left(\begin{array}{l}b \\ 0\end{array}\right)=1,\left(\begin{array}{l}0 \\ i\end{array}\right)=0$ for $i>0$, and $\left(\begin{array}{c}b+1 \\ i\end{array}\right)=\left(\begin{array}{c}b \\ i\end{array}\right)+\left(\begin{array}{c}b \\ i-1\end{array}\right)$.

We will now confirm that there are no other stable operations.

THEOREM (2.7). The operations discussed above are all distinct, and provide all of the stable operations of Borel cohomology; indeed $b^{*} b=b^{*} \tilde{\otimes} A^{*}$.

PROOF. To see that no nontrivial element of $b^{*} \tilde{\otimes} A^{*}$ acts trivially on cohomology we consider the same $G$-fixed spaces $X$ as are used to show the analogous fact for $A^{*}$ (products of $E M\left(\mathbf{F}_{p}, 1\right)$ are convenient [26]) and evaluate on the classes $1 \otimes x \in b^{*} \otimes H^{*} X=b^{*} X$. It follows that all the operations arising from $b^{*} \tilde{\otimes} A^{*}$ are distinct.

It remains to verify that we have formed all stable operations, and for this we verify that $b^{n} b$ has the same dimension as the subspace $\left(b^{*} \tilde{\otimes} A^{*}\right)^{n}$. Now we have 
$b \simeq \operatorname{holim}_{k} S^{-k \rho} E M\left(\mathbf{F}_{p}, k|\rho|\right)^{E G_{+}}$as remarked above, and hence a Milnor exact sequence for calculating $b^{n} b$. The following is the fundamental lemma that allows us to proceed.

LemMA 2.8. For any $G$-space $X$ the collapse map $\pi: E G_{+} \rightarrow S^{0}$ induces an equivalence

$$
1^{\pi} \wedge 1: X^{S^{0}} \wedge E G_{+} \rightarrow X^{E G_{+}} \wedge E G_{+} .
$$

In fact evaluation induces an inverse.

ProOF. Of course $\pi$ is a nonequivariant equivalence, and so the same is true of $1^{\pi} \wedge 1$. Since both domain and codomain are $G$-free this is enough to establish that $1^{\pi} \wedge 1$ is an equivalence. It is clear that evaluation defines a left inverse to $1^{\pi} \wedge 1$, and it follows that it is also a right inverse.

We therefore find in particular that if $X$ is $G$-fixed

$$
b^{*}\left(X^{E G_{+}}\right)=b^{*}\left(X^{E G_{+}} \wedge E G_{+}\right)=b^{*}\left(X \wedge E G_{+}\right)=b^{*} \otimes H^{*} X .
$$

Note that if $X=E M\left(\mathbf{F}_{p}, k|\rho|\right)$ this group is finite in each degree and hence the Rlim term of the Milnor exact sequence vanishes to give

$$
b^{n} b=\lim _{k}\left\{b^{*} \otimes H^{*}\left(E M\left(\mathbf{F}_{p}, k|\rho|\right)\right)\right\}^{n+k|\rho|} .
$$

Since the dimension of $b^{*} \otimes H^{*}\left(E M\left(\mathbf{F}_{p}, k|\rho|\right)\right)$ in degree $n+k|\rho|$ is constant for large $k$ and equal to that of $\left\{b^{*} \tilde{\otimes} A^{*}\right\}^{n}$ it follows that $\left\{b^{*} \tilde{\otimes} A^{*}\right\}^{n}$ exhausts $b^{n} b$. (It also follows that the maps of the inverse system are eventually isomorphic.) This completes the proof of (2.7).

3. The construction of the spectral sequence. We continue to suppose that $p=2$ if $G$ is even order.

In this section we will construct an Adams spectral sequence based on co-Borel cohomology under the assumption that $Y$ is $G$-free and $b^{*} Y$ is locally finite and bounded below. These assumptions are obviously satisfied if conditions (i), (iii) and (iv) of Theorem 0 hold. Its $E_{2}$ term takes the usual form (3.4), and in the next section we show the spectral sequence is convergent under the further assumption that $Y$ is bounded below and $p$-complete.

Since $b^{*} Y$ and $b^{*} b$ are locally finite and bounded below, it follows that we may form an algebraic resolution of $b^{*} Y$ by locally finite bounded below free $b^{*} b$-modules

$$
\cdots \rightarrow P_{2} \stackrel{\partial_{2}}{\rightarrow} P_{1} \stackrel{\partial_{1}}{\rightarrow} P_{0} \stackrel{\varepsilon}{\rightarrow} b^{*} Y \rightarrow 0
$$

We now want to realize (3.1) geometrically and for this purpose we need to know that certain sums and products coincide.

LEMMA (3.2). If $\left\{n_{j}\right\}_{j \geq 0}$ is a sequence of integers such that $n_{j} \rightarrow \infty$ as $j \rightarrow \infty$ then

(a) the natural algebraic map $\nu: \bigoplus_{j} c^{*}\left(S^{n_{j}} c\right) \rightarrow \prod_{j} c^{*}\left(S^{n_{j}} c\right)$ is an isomorphism and

(b) the natural geometric map $\nu: \bigvee_{j} S^{n_{j}} c \rightarrow \prod_{j} S^{n_{j}} c$ is an equivalence.

PROOF. (a) The algebraic statement follows immediately from the fact that $c^{*} c$ is bounded below and locally finite. 
(b) The geometric fact relies on the fact that $c_{*}\left(G / H_{+}\right)=H_{*}\left(B H_{+}\right)$is bounded below and hence for each subgroup $H$ and integer $r$ only finitely many of the groups $\left[G / H_{+}, S^{n_{j}} c\right]_{r}^{G}$ are nonzero.

It follows that the map $\nu$ induces an isomorphism of $\left[G / H_{+}, \cdot\right]_{*}^{G}$ for all $H$, and hence that $\nu$ is an equivalence by the Whitehead theorem.

REMARK. The statement analogous to $(3.2)(\mathrm{b})$ with $c$ replaced by $b$ is false; this construction of the spectral sequence therefore forces us to use co-Borel cohomology.

Now since $b^{*} b=b^{*} c$, by $(3.2)\left(\right.$ a) we may construct a spectrum $Q_{s}$ so that $P_{s} \cong b^{*} S^{s} Q_{s}$ by taking $Q_{s}$ to be a locally finite wedge of suspensions of $c$.

By $(3.2)(\mathrm{b}) \varepsilon$ is induced by a map $Y_{0} \rightarrow Q_{0}$ and we may proceed as usual to realise the algebraic resolution (3.1) as an Adams tower:

$$
\begin{array}{ccccc}
S^{-1} Q_{1} & \rightarrow & \begin{array}{c}
\downarrow \\
Y_{2}
\end{array} & \rightarrow & Q_{2} \\
& & \downarrow & & \\
S^{-1} Q_{0} & \rightarrow & Y_{1} & \rightarrow & Q_{1} \\
& & \downarrow \\
& & & \\
& & Y_{0} & \rightarrow & Q_{0}
\end{array}
$$

Here $Y_{s+1}$ is constructed as the fibre of a map $Y_{s} \rightarrow Q_{s}$ inducing the map $\partial_{s}$ of $P_{s}$ onto the kernel of $\partial_{s-1}$. Accordingly $Y_{s+1}$ is $G$-free.

It also follows as usual that an algebraic comparison of resolutions (3.1) over a map $f^{*}: b^{*} Y \rightarrow b^{*} Y^{\prime}$ induced by a map $f: Y^{\prime} \rightarrow Y$ may be realised uniquely as a map of towers (3.3).

We now apply $[X,]^{G}$ to $(3.3)$ and obtain a spectral sequence with $E_{1}^{s, t}=$ $\left[S^{t-s} X, Q_{s}\right]^{G}$ which is conditionally convergent to $\left[S^{t-s} X, Y / \operatorname{holim}_{s} Y_{s}\right]^{G}[\mathbf{5}]$.

COROLlARY (3.4). The spectral sequence described above has the $E_{2}$ term naturally identified by

$$
E_{2}^{s, t}=\operatorname{Ext}_{c^{*}{ }^{*}}^{s, t}\left(c^{*} Y, c^{*} X\right) \text {. }
$$

ProOF. We have

$$
\begin{aligned}
E_{1}^{s, t} & =\left[S^{t} X, S^{s} Q_{s}\right]^{G} \\
& =\left[S^{t} X, \prod_{j} S^{n_{j}} c\right]^{G} \quad(\text { by }(3.2)(\mathrm{b})) . \\
& =\prod_{j}\left[S^{t} X, S^{n_{j}} c\right]^{G}=\prod_{j} c^{n_{j}}\left(S^{t} X\right) \\
& =\operatorname{Hom}_{c^{*} c}^{t}\left(\bigoplus_{j} c^{*}\left(S^{n_{j}} c\right), c^{*} X\right) \\
& =\operatorname{Hom}_{c^{*}{ }^{t}}\left(c^{*} S^{s} Q_{s}, c^{*} X\right) \quad(\mathrm{by}(3.2)(\mathrm{a})) .
\end{aligned}
$$

Since these identifications are natural the description of $E_{2}$ follows. The naturality of the $E_{2}$-term in $Y$ comes from the fact that there is a map of towers over any map $Y \rightarrow Y^{\prime}$. Naturality in $X$ is clear.

4. Convergence. In this section we assume that $G$ is a $p$-group and $k=\mathbf{F}_{\boldsymbol{p}}$.

We will prove that the Adams spectral sequence based on co-Borel cohomology constructed in $\S 3$ is convergent under mild completeness assumptions. 
THEOREM (4.1). If $Y$ is $G$-free, bounded below and $p$-complete, and if $b^{*} Y$ is locally finite then

$$
\operatorname{holim}_{s} Y_{s} \simeq *
$$

REMARKS. (1) It already follows from the convergence of the spectral sequence to $\left[X, Y / \operatorname{holim}_{s} Y_{s}\right]_{*}^{G}$ that the homotopy type of $\operatorname{holim}_{s} Y_{s}$ is independent of the resolution (3.1) used to construct the tower.

(2) The two main ingredients of the proof are the fact that since $G$ is a $p$-group the only simple $\mathbf{F}_{p} G$-module is $G$-trivial, and the fact that $b^{*} b$ is a connected $\mathbf{F}_{p^{-}}$ algebra.

PROOF. By Remark (1) above and since $b^{*} b$ is connected, we may suppose that the resolution $(3.1)$ is minimal in the sense that connectivity $\left(P_{s}\right)=$ connectivity $\left(\operatorname{ker} \partial_{s}\right)$ and the map $P_{s} \rightarrow \operatorname{ker} \partial_{s}$ is isomorphic in this codegree. It follows also that

$$
\operatorname{connectivity}\left(P_{s}\right) \geq \operatorname{connectivity}\left(b^{*} Y\right)+s .
$$

The first step of the proof is to verify that this implies that the connectivities of the $Y_{s}$ are uniformly bounded below (by the connectivity of $Y$ ).

LEMMA (4.2). If $G$ is a p-group and we use $\bmod p$ coefficients and if $Z$ is bounded below then

$$
\text { connectivity }\left(b^{*} Z\right)=\operatorname{connectivity}\left(H^{*} Z\right) .
$$

ProOF. Filtering $E G_{+}$by skeleta, we obtain a spectral sequence

$$
H^{*}\left(G ; H^{*} Z\right) \Rightarrow b^{*} Z
$$

which is (strongly) convergent since $Z$ is bounded below.

Now if $H^{*} Z$ is precisely $m$-connected we have $b^{*} Z m$-connected and $b^{m+1} Z=$ $H^{0}\left(G ; H^{m+1} Z\right)$; since $G$ is a $p$-group and $H^{m+1} Z$ is a nonzero $\mathbf{F}_{p} G$-module, $b^{m+1} Z \neq 0$. Hence $b^{*} Z$ is also precisely $m$-connected.

REMARK. This lemma is the only reason we must restrict $G$ to be a $p$-group.

COROLlaRY (4.3). The connectivities of the $Y_{s}$ are uniformly bounded below by the connectivity of $Y$.

PrOOF. Since $Y_{s}$ is $G$-free its equivariant connectivity is the same as its nonequivariant connectivity. Since $Y_{s}$ is bounded below this connectivity is that of its integral homology, and since it is $p$-complete this is the connectivity of $H^{*}\left(Y_{s}\right)$ (i.e. with $\bmod p$ coefficients).

By (4.2) it therefore suffices to verify that connectivity $\left(c^{*}\left(Y_{s}\right)\right) \geq$ connectivity $\left(c^{*} Y\right)$. However, by construction $c^{*}\left(Y_{s}\right)=S^{-s} \operatorname{ker}\left(\partial_{s-1}\right)$, and by minimality

$$
\text { connectivity }\left(\operatorname{ker}\left(\partial_{s-1}\right)\right)=\operatorname{connectivity}\left(P_{s}\right) \geq \operatorname{connectivity}\left(b^{*} Y\right)+s .
$$

The next step is to use the following lemma to deduce from (4.3) that $\operatorname{holim}_{s} Y_{s}$ is equivalent to a $G$-free spectrum. We may then use nonequivariant results to deduce that $\operatorname{holim}_{s} Y_{s}$ is contractible. 
LEMMA (4.4). If $\left(Z_{\alpha}\right)_{\alpha \in A}$ is a uniformly bounded below collection of spectra then the natural map

$$
\nu: E G_{+} \wedge \prod_{\alpha} Z_{\alpha} \rightarrow \prod_{\alpha} E G_{+} \wedge Z_{\alpha}
$$

is an equivalence.

PrOOF. We will show that $\nu$ induces an isomorphism of $[T, \cdot]^{G}$ for any finite $T$. This is sufficient by the Whitehead theorem.

Now if $Z_{\alpha}$ is $c$-connected for all $\alpha, \prod_{\alpha} Z_{\alpha}$ is also $c$-connected; hence the two horizontals in the following diagram are isomorphic for $n>c+\operatorname{dimension}(T)$

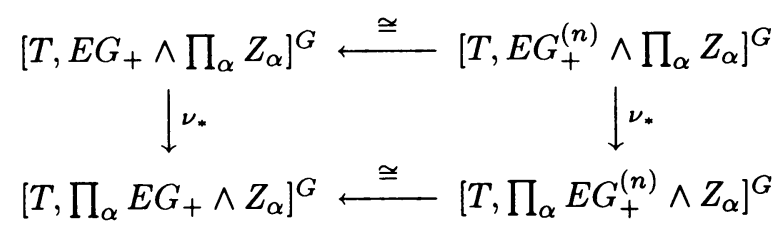

Since $E G_{+}^{(n)}$ is a finite complex, the right-hand vertical is easily seen to be isomorphic (for example because $E G_{+}^{(n)}$ is its own Spanier-Whitehead double dual).

COROLlARY (4.5). If $\left(Z_{\alpha}\right)_{\alpha \in A}$ is a uniformly bounded below collection of spectra then the natural map

$$
\nu: c \wedge \prod_{\alpha} Z_{\alpha} \rightarrow \prod_{\alpha} c \wedge Z_{\alpha}
$$

is an equivalence.

PROOF. By (4.4) it is enough to verify that $\nu$ is nonequivariantly an equivalence. But $c$ is nonequivariantly the Eilenberg-Mac Lane spectrum so the result follows from Adams' powerful Theorem (15.2) of [2].

Accordingly we have

$$
c \wedge \operatorname{holim}_{s} Y_{s} \simeq \operatorname{holim}_{s} c \wedge Y_{s}
$$

and hence a short exact sequence

$$
0 \rightarrow \operatorname{Rlim}_{s} c_{*} S^{-1} Y_{s} \rightarrow c_{*} \operatorname{holim}_{s} Y_{s} \rightarrow \lim _{s} c_{*} Y_{s} \rightarrow 0 .
$$

Furthermore since the $Y_{s}$ are $G$-free their co-Borel homology and cohomology are $\mathbf{F}_{p}$-dual to each other. Hence the maps $Y_{s+1} \rightarrow Y_{s}$ induce zero in $c_{*}(\cdot)$ as well as $c^{*}(\cdot)$ and therefore $c_{*} \operatorname{holim}_{s} Y_{s}=0$. It follows that $b^{*} \operatorname{holim}_{s} Y_{s}=0$, and since $\operatorname{holim}_{s} Y_{s}$ is bounded below $H^{*} \operatorname{holim}_{s} Y_{s}=0$ by (4.2). But $\operatorname{holim}_{s} Y_{s}$ is $G$-free, $p$-complete and bounded below and so we deduce that holim ${ }_{s} \longleftarrow \simeq$ as required. This completes the proof of (4.1).

In the proof of (4.1) the major step was to establish that a product of $G$-free spectra is $G$-free if the spectra are uniformly bounded below. We conclude with an example to show that a product of arbitrary $G$-free spectra need not be $G$-free.

EXAMPLE (4.6). Suppose $G$ is cyclic of order 2 and $\xi$ is the nontrivial 1dimensional real representation, and consider the inverse system

$$
\cdots \rightarrow S^{k \xi} c \rightarrow S^{(k+1) \xi_{c}} \rightarrow S^{(k+2) \xi} c \rightarrow \cdots .
$$


If $X=\operatorname{holim}_{k} S^{k \xi} c$ then
(a) $X \not{\not} *$,
(b) $X$ is nonequivariantly contractible, and
(c) $X$ is not $G$-free.

PROOF. Part (b) is clear since the inclusions $S^{k \xi} \rightarrow S^{(k+1) \xi}$ are nonequivariantly null, and (c) follows from (a) and (b).

For (a) we note that

$$
\left[S^{r}, \operatorname{holim}_{k} S^{k \xi} c\right]^{G}=\lim _{k} c_{k \xi}\left(S^{r}\right)
$$

and that the maps of the inverse system are multiplication by the Euler class. Since multiplication by the Euler class is isomorphic for $k>r$ it follows that the above group is $\mathbf{F}_{2}$ for all $r$.

REMARK (4.7). The previous example is a special case of the fact that the Bousfield completion of the co-Borel spectrum $c$ with respect to nonequivariant homology is the Borel spectrum $b[\mathbf{1 5}]$.

5. Applications. In this section we will show how certain general geometric facts about maps into free spectra are reflected in the algebraic properties of coBorel cohomology. Most interesting of these are the connection (5.8) of co-Borel cohomology with the Singer construction [24] and the injectivity of $c^{*}$ as a $b^{*}$ module.

The most primitive application of the spectral sequence is a slight generalisation of theorems of Carlsson [8, Theorem B(a)] and May [21, Theorem 13] in view of Serre's theorem on the nilpotence of certain Euler classes [23].

THEOREM (5.1). Suppose that $Y$ satisfies the condition of Theorem 0 . If some power of the Euler class $e(V)$ acts trivially on $c^{*}(X)$ then $\left[S^{\infty V} \wedge X, Y\right]_{*}^{G}=0$.

PROOF. Under the hypotheses of the theorem $c^{*}\left(S^{\infty V} \wedge X\right)=0$ and so the $c^{*} c$ spectral sequence has zero $E_{1}$-term.

We now turn to slightly more sophisticated uses of the spectral sequence, which are based on algebraic methods of disentangling equivariant and topological features in the $E_{2}$-term. These are summarised in the following lemma.

For this we suppose $M$ and $N$ are left $b^{*} \tilde{\otimes} A^{*}$-modules and $L$ is a left $A^{*}$-module; we make $M \otimes L$ into a left $b^{*} \tilde{\otimes} A^{*}$-module by using the diagonal $A^{*}$-action, and we make $\operatorname{Hom}_{b^{*}}(M, N)$ into a left $A^{*}$-module using Thom's canonical antiautomorphism $\chi$ in the formula

$$
(a f)(x):=\sum_{i}(-1)^{\left|a_{i}^{\prime \prime}\right||f|} a_{i}^{\prime} f\left(\chi\left(a_{i}^{\prime \prime}\right) x\right)
$$

where $a \in A^{*}, f \in \operatorname{Hom}_{b^{*}}(M, N), x \in M$ and where $\psi^{*} a=\sum_{i} a_{i}^{\prime} \otimes a_{i}^{\prime \prime}$ is the diagonal expansion of $a$. We leave the reader to verify that $a f$ is a $b^{*}$-map.

LEMMA (5.2). With $L, M$ and $N$ as above we have a natural isomorphism

$$
\operatorname{Hom}_{A^{*}}\left(L, \operatorname{Hom}_{b^{*}}(M, N)\right)=\operatorname{Hom}_{b^{*} \tilde{\otimes} A^{*}}(M \otimes L, N)
$$

and hence a composite functor spectral sequence

$$
E_{2}^{\sigma, \tau}=\operatorname{Ext}_{A^{*}}^{\sigma}\left(L, \operatorname{Ext}_{b^{*}}^{\tau}(M, N)\right) \Rightarrow \operatorname{Ext}_{b^{*} \tilde{\otimes} A^{*}}^{\sigma+\tau}(M \otimes L, N)
$$

The proof of the first part is a routine verification, and the second part follows by standard methods $[\mathbf{1 0}]$. 
COROLlaRY (5.3). (1) There is a spectral sequence

$$
E_{2}^{\sigma, \tau}=\operatorname{Ext}_{A^{*}}^{\sigma}\left(\mathbf{F}_{p}, \operatorname{Ext}_{b^{*}}^{\tau}\left(b^{*} Y, c^{*} X\right)\right) \Rightarrow \operatorname{Ext}_{b^{*} \tilde{\otimes} A^{*}}^{\sigma+\tau}\left(b^{*} Y, c^{*} X\right)
$$

converging to the $E_{2}$-term of the $c^{*} c$ spectral sequence.

(2) If $b^{*} Y$ is $b^{*}$-projective then the $c^{*} c$ spectral sequence has $E_{2}$-term

$$
E_{2}^{s, t}=\operatorname{Ext}_{A^{*}}^{s, t}\left(\mathbf{F}_{p} \otimes_{b^{*}} b^{*} Y, c^{*} X\right) .
$$

(3) If $c^{*} X$ is $b^{*}$-injective then the $c^{*} c$ spectral sequences has $E_{2}$-term

$$
E_{2}^{s, t}=\operatorname{Ext}_{A^{*}}^{s, t}\left(\mathbf{F}_{p}, \operatorname{Hom}_{b^{*}}\left(b^{*} Y, c^{*} X\right)\right) .
$$

Of course a notable case of interest where $b^{*} Y$ is $b^{*}$-projective is when $Y=Y^{\prime} \wedge$ $E G_{+}$and $Y^{\prime}$ is a $G$-fixed space so that $b^{*} Y=b^{*} \tilde{\otimes} H^{*} Y$. In fact the corresponding case when $X$ is a $G$-fixed space, so that $c^{*} X=c^{*} \tilde{\otimes} H^{*} X$ is relevant to (3) by the next lemma. Indeed this sheds some light on Adams' isomorphism $[X, Y / G]^{1}=[X, Y]^{G}$ when $Y$ is $G$-free and $X$ is $G$-fixed.

Lemma (5.4). The $b^{*}$-module $c^{*}$ is injective.

PROOF. Since cap product is dual to cup product we have

$$
c^{*}=\operatorname{Hom}_{\mathbf{F}_{p}}^{*}\left(b^{*}, \mathbf{F}_{p}\right) .
$$

Since $\mathbf{F}_{p}$ is $\mathbf{F}_{p}$-injective the usual argument that $\operatorname{Hom}_{\mathbf{Z}}(R, \mathbf{Q} / \mathbf{Z})$ is $R$-injective applies to allow us to conclude $c^{*}$ is $b^{*}$-injective.

We now return to the case when $Y$ is $b^{*}$-projective. In view of the resemblance of the $E_{2}$-term to the $E_{2}$-term of a nonequivariant Adams spectral sequence we may hope for a geometric corollary of (5.3)(2), and the following is a useful tool.

Proposition (5.5). If $b^{*} Y$ is $b^{*}$-projective then the $c$ Adams tower (3.3) is nonequivariantly an $H$ Adams tower.

The proof of this is based on an interesting lemma.

Lemma (5.6). Suppose $G$ is a p-group and $k=\mathbf{F}_{p}$. If $b^{*} Y$ is $b^{*}$-free and $Y$ is bounded below then

(a) $H^{*} Y$ is a $G$-fixed $\mathbf{F}_{p} G$-module,

(b) the spectral sequence $H^{*}\left(G ; H^{*} Y\right) \Rightarrow b^{*} Y$ collapses, and

(c) $b^{*} Y \cong b^{*} \tilde{\otimes} H^{*} Y$ as $b^{*} b$-modules.

PROOF. We argue row by row in the spectral sequence, working upwards. Indeed if $Y$ is $(c-1)$-connected, let $M=H^{c}(Y)$. Clearly $b^{c}(Y)=H^{0}(G ; M)$ and hence the composite

$$
H^{*}\left(G ; M^{G}\right) \rightarrow H^{*}(G ; M) \rightarrow E_{\infty}^{*, c} \rightarrow b^{*}(Y)
$$

is injective in codegree $c$. Since it is a $b^{*}$-map and $b^{*} Y$ is $b^{*}$ free the composite is injective. Hence in particular the map $i: M^{G} \rightarrow M$ of coefficient modules is injective in $H^{*}(G ; \cdot)$. Since $i$ is tautologically surjective in $H^{0}(G ; \cdot)$ it follows that $H^{0}\left(G ; M / M^{G}\right)=0$ and hence (since $G$ is a $p$-group) that $M / M^{G}=0$. Thus $M$ is a $G$-fixed $\mathbf{F}_{p} G$-module and therefore

$$
H^{*}(G ; M) \rightarrow b^{*} Y
$$


is injective, so that no differentials arrive on the bottom row. Since $H^{*}(G ; M)$ is $b^{*}$-generated by $H^{0}(G ; M)$ it follows that $b^{*} Y / H^{*}(G ; M)$ is still $b^{*}$-free. We may now repeat this argument for higher rows.

This completes the proof of (a) and (b).

Part (c) follows provided we can check that the quotient $H^{*} Y$ of $b^{*} Y$ has the usual $A^{*}$-action. This follows since the quotient is induced by $E G_{+}^{(0)} \wedge Y \rightarrow E G_{+} \wedge Y$ in $b^{*}(\cdot)$.

REMARK. Part (a) of the above lemma is a slight generalisation of the fact that if $G$ is a $p$-group and $H^{*}(G ; M)$ is free over $H^{*}(G)$ then $M$ is $G$-fixed.

PROOF OF (5.5). Since $c$ is nonequivariantly the Eilenberg-Mac Lane spectrum $H$, we need only show that the maps $Y_{s} \rightarrow Q_{s}$ induce $H^{*}(\cdot)$-epimorphisms. By definition of projectivity we find that the resolution (3.1) of $b^{*} Y$ consists of $b^{*}$ projectives and that the kernels of $\partial_{s}$ are $b^{*}$-projective. Hence $b^{*} Y_{s}$ and $b^{*} Q_{s}$ are $b^{*}$-projective. Now the required surjectivity follows from (5.6).

We now turn to another class of $X$ for which $c^{*} X$ is $b^{*}$-injective. In fact we are lead to a connection with the Singer construction and other algebra that has already proved important in equivariant topology. First we need a lemma.

LEMMA (5.7). For any $X$ we have a natural isomorphism

$$
c^{*}(X)=\operatorname{Hom}_{\mathbf{F}_{p}}^{*}\left(b_{*} X, \mathbf{F}_{p}\right)
$$

of $b^{*} b$-modules.

PROOF. We have natural transformations of $b^{*} b$-modules

$$
c^{*}(X) \rightarrow \operatorname{Hom}_{b_{*}}^{*}\left(b_{*} X, c_{*}\right) \rightarrow \operatorname{Hom}_{\mathbf{F}_{p}}^{*}\left(b_{*} X, \mathbf{F}_{p}\right)
$$

induced by the $b$-module structure of $c$ and the algebraic augmentation map $c_{*} \rightarrow$ $\mathbf{F}_{p}$. If $X=D Y$ is the dual of a finite complex the composite gives the cap isomorphism

$$
H_{*}\left(E G_{+} \wedge_{G} Y\right) \stackrel{\cong}{\rightrightarrows} \operatorname{Hom}_{\mathbf{F}_{p}}^{*}\left(H^{*}\left(E G_{+} \wedge_{G} Y\right), \mathbf{F}_{p}\right) .
$$

Since both source and target are cohomology theories the lemma follows.

We may now establish the connection with the Singer construction $R_{+} M[\mathbf{2 4}]$ in the case $G=C_{p}$. It is based on H. Miller's isomorphism [7, II.5.1].

COROllary (5.8). Suppose $G=C_{p}$ and $Y$ is a $G$-fixed space or spectrum. We have a natural isomorphism of $b^{*} b$-modules

$$
b_{*}\left(S^{\infty \tilde{\rho}} \wedge Y\right)=S_{-1} R_{+} H_{*} Y
$$

and hence also

$$
c^{*}\left(S^{\infty \tilde{\rho}} \wedge Y\right)=S^{-1}\left\{R_{+} H_{*} Y\right\}^{*}
$$

ProOF. Since $b_{*}(\cdot), H_{*}(\cdot)$ and $R_{+}(\cdot)$ preserve exactness and direct limits it suffices to construct a natural isomorphism when $Y$ is finite. Since $R_{+}(\cdot)$ is stable we may even suppose that $Y=D Z$ for some space $Z$. 
Now we use a short calculation in which $D$ denotes Spanier-Whitehead duality and $D_{p}$ denotes the extended power construction [7].

$$
\begin{aligned}
b_{*}\left(S^{\infty \tilde{\rho}} \wedge D Z\right) & =b_{*}\left(S^{\infty \tilde{\rho}} \wedge D\left(Z^{\wedge p}\right)\right) \quad \begin{array}{r}
\text { (since passage to fixed points commutes } \\
\text { with Spanier-Whitehead duality). }
\end{array} \\
& =\underset{\vec{k}}{\lim } b_{*}\left(S^{k \tilde{\rho}} \wedge D\left(Z^{\wedge p}\right)\right) \\
& =\underset{k}{\lim } b^{*}\left(S^{-k \tilde{\rho}} \wedge Z^{\wedge p}\right) \\
& =\underset{k}{\lim } b^{*}\left(S^{k}\left(S^{-k} Z\right)^{\wedge p}\right) \\
& =\frac{\lim _{k}}{\longrightarrow} H^{*}\left(S^{k} D_{p} S^{-k} Z\right) \\
& =S^{-1} R_{+} H^{*} Z \quad \text { (by Miller's isomorphism). }
\end{aligned}
$$

The fundamental property of the Singer construction is the fact that there is an augmentation of degree zero

$$
\varepsilon: R_{+} M \rightarrow M
$$

which induces an isomorphism of $\operatorname{Ext}_{A^{*}}(\cdot, N)$ provided $N$ admits a finite filtration by $A^{*}$-submodules with $A^{*}$-trivial subquotients. Consequently its dual $\varepsilon^{*}$ induces an isomorphism of $\operatorname{Ext}_{A^{*}}(N, \cdot)$ under the same hypotheses.

For a more general elementary abelian group $G$ of rank $n$ Adams-GunawardenaMiller [4] shows that the quotient map

$$
\varepsilon^{\prime}: b_{*}\left(S^{\infty \tilde{\rho}}\right) \rightarrow \mathbf{F}_{p} \otimes_{A^{*}} b_{*}\left(S^{\infty \tilde{\rho}}\right)
$$

also induces isomorphisms of the above Ext groups. However it is not known if $b_{*}\left(S^{\infty \tilde{\rho}} \wedge Y\right)$ is generally a functor of the $A^{*}$-module $H_{*} Y$, nor is there a known transformation to $S_{-n} H_{*} Y$.

COROLlaRY (5.9). Suppose $b^{*} Y$ is $b^{*}$-projective and $H^{*} Y$ admits a finite $A^{*}$-filtration with $A^{*}$-trivial subquotients. We have the following description of $E_{2}^{s, t}=\operatorname{Ext}_{c^{*} c}^{s, t}\left(b^{*} Y, c^{*}\left(S^{\infty \tilde{\rho}} \wedge X^{\prime}\right)\right)$ for $G$-fixed $X^{\prime}$.

(a) If $G=C_{p}, E_{2}^{s, t} \cong \operatorname{Ext}_{A^{*}}^{s, t}\left(H^{*} Y, S^{-1} H^{*} X^{\prime}\right)$.

(b) If $G$ is elementary abelian and $X^{\prime}=S^{0}$ then

$$
E_{2}^{s, t}=\operatorname{Ext}_{A^{*}}^{s, t}\left(H^{*} Y, S^{-n}\left(S t^{*}\right)\right),
$$

where $S t^{*}$ is a vector space of dimension $n(n-1) / 2$ in codegree 0 .

This has clear connections with theorems of May [21, 9]. Geometric corollaries in the elementary abelian case are best discussed in the context of [14].

Finally we have a version of the fact that all maps from a nonequivariantly contractible space to a finite $G$-free space are null.

THEOREM (5.10). Suppose $Y$ satisfies the conditions of Theorem 0 . If

(i) $b^{*} Y$ admits a finite filtration by $b^{*} b$ submodules with $b^{*}$-trivial subquotients, and

(ii) $X$ is nonequivariantly contractible

then $[X, Y]_{*}^{G}=0$. 
ProOF. Of course since $X$ is nonequivariantly contractible we find $X \simeq X \wedge$ $\left(S^{0} * E G\right)$, and by the Milnor exact sequence it is sufficient to consider the case $X=G / H_{+} \wedge S^{0} * E G$.

Since $G$ is a $p$-group $S^{0} * E G$ can be constructed from infinite spheres $S^{\infty V}$ for various representations $V$. Accordingly it is enough to show

$$
\left[G / H_{+} \wedge S^{\infty V}, Y\right]_{*}^{G}=0
$$

We will do this by showing that the $E_{2}$-term of the spectral sequence of $(5.3)(1)$ is zero, and hence that the $E_{2}$ term of the $c^{*} c$ spectral sequence is zero. By the hypothesis (i) it is enough to prove the following.

LEMMA (5.11). $\operatorname{Ext}_{b^{*}}^{s}\left(\mathbf{F}_{p}, c^{*}\left(G / H_{+} \wedge S^{\infty V}\right)\right)=0$ for all $s$.

PROOF. For this we deduce from (5.7) that

$$
c^{*}\left(G / H_{+} \wedge S^{\infty V}\right) \cong\left\{H^{*} B H_{+}\left[e(V)^{-1}\right]\right\}^{*}
$$

Now $H^{*} B H_{+}$may be resolved by $b^{*}$-free modules, and hence we obtain a flat resolution of $H^{*} B H_{+}\left[e(V)^{-1}\right]$ and thereby an injective resolution of $c^{*}\left(G / H_{+} \wedge\right.$ $\left.S^{\infty V}\right)$ using modules of form $\left\{M\left[e(V)^{-1}\right]\right\}^{*}$.

Now we need only observe that

$$
\operatorname{Hom}_{b^{*}}\left(\mathbf{F}_{p},\left\{M\left[e(V)^{-1}\right]\right\}^{*}\right)=0
$$

Indeed $\left\{M\left[e(V)^{-1}\right]\right\}^{*}$ is either zero or $e(V)^{*}$-local.

This completes the proof of $(5.10)$.

\section{REFERENCES}

1. J. F. Adams, On the structure and applications of the Steenrod algebra, Comment. Math. Helv. 32 (1958), 180-214.

2. 1974.

3. _ Prerequisites (on equivariant stable homotopy) for Carlsson's lecture, Lecture Notes in Math., vol. 1051, Springer-Verlag, 1984, pp. 483-532.

4. J. F. Adams, J. H. C. Gunawardena and H. R. Miller, The Segal conjecture for elementary abelian p-groups, Topology 24 (1985), 435-460.

5. J. M. Boardman, Conditionally convergent spectral sequences, Preprint, The John Hopkins University, August 1981.

6. A. Borel, Seminar on transformation groups, Chapter IV, Princeton Univ. Press, Princeton, N. J., 1960.

7. R. R. Bruner, J. P. May, J. E. McClure and M. Steinberger, $H_{\infty}$ ring spectra and their applications, Lecture Notes in Math., vol. 1176, Springer-Verlag, 1986.

8. G. Carlsson, Equivariant stable homotopy and Segal's Burnside ring conjecture, Ann. of Math. (2) 120 (1984), 189-224.

9. J. Caruso, J. P. May and S. B. Priddy, The Segal conjecture for elementary abelian p-groups II; p-adic completion in equivariant cohomology, Topology 26 (1987), 413-433.

10. H. Cartan and S. Eilenberg, Homological algebra, Princeton Univ. Press, 1956.

11. J. P. C. Greenlees, Representing Tate cohomology of G-spaces, Proc. Edinburgh Math. Soc. 30 (1987), 435-443.

12. _ Topological methods in equivariant cohomology, Proc. 1987 Singapore Group Theory Conference, De Gruyter (to appear).

13. __ Borel homology I, Preprint (1987). 
14. __ Borel homology II (in preparation).

15. Homotopy equivariance strict equivariance and the Segal conjecture (in preparation).

16. L. G. Lewis, J. P. May and J. McClure, Ordinary $R O(G)$-graded cohomology, Bull. Amer. Math. Soc. (N.S.) 4 (1981), 208-212.

17. L. G. Lewis, J. P. May, J. McClure and S. Waner, Equivariant cohomology theory (in preparation).

18. L. G. Lewis, J. P. May and M. Steinberger (with contributions by J. E. McClure), Equivariant stable homotopy theory, Lecture Notes in Math., vol. 1213, Springer-Verlag, 1986.

19. W. H. Lin, On conjectures of Mahowald, Segal and Sullivan, Math. Proc. Cambridge Philos. Soc. 87 (1980), 449-458.

20. W. S. Massey and F. P. Peterson, The cohomology structure of certain fibre spaces I, Topology 4 (1965), 47-65.

21. J. P. May, The completion conjecture in equivariant cohomology, Lecture Notes in Math., vol. 1051, Springer-Verlag, 1984, pp. 620-637.

22. J. W. Milnor, The Steenrod algebra and its dual, Ann. of Math. (2) 67 (1958), 150-171.

23. J.-P. Serre, Sur la dimension cohomologique des groupes profinis, Topology 3 (1965), 413-420.

24. W. M. Singer, A new chain complex for the homology of the Steenrod algebra, Math. Proc. Cambridge Philos. Soc. 90 (1981), 279-292.

25. N. E. Steenrod, A convenient category of topological spaces, Michigan Math. J. 14 (1967), 133-152.

26. N. E. Steenrod and D. B. A. Epstein, Cohomology operations, Princeton Univ. Press, 1962.

27. R. G. Swan, A new method in fixed point theory, Comment. Math. Helv. 34 (1960), 1-16.

28. S. Waner, Equivariant $R O(G)$-graded singular cohomology, Preprint (1979).

29. G. W. Whitehead, Generalised homology theories, Trans. Amer. Math. Soc. 102 (1962), 227283.

Department of Mathematics, National University of Singapore, Kent RIDGE, SINGAPORE 0511 